\title{
Spatial problem solving in the rat following medial frontal lesions
}

\author{
THOM HERRMANN \\ University of Guelph, Guelph, Ontario, Canada \\ BRUNO POUCET \\ C.N.R.S., Marseille, France \\ and \\ PAUL ELLEN \\ Georgia State University, Atlanta, Georgia
}

\begin{abstract}
Rats with medial frontal lesions were unable to perform as successfully as normal rats on the Maier 3-table spatial reasoning task. Performance did not improve with repeated testing. Instead, the test-trial performance of medial frontal animals was characterized by the use of a directional response (i.e., body turn) strategy at the choice point. Normal rats habituated (i.e., decreased the number of table entries) within the first few minutes of the daily exploratory period. Although medial frontal rats initially did not display this habituation pattern, they did develop this habituation pattern over blocks of trials. The presence of the habituation pattern has been used as evidence to indicate that the table-entry behavior reflects exploratory rather than general activity levels. The fact that medial frontal animals do show a habituation pattern but are unable to solve the problem suggests that such animals are able to acquire spatial information but are unable to use it.
\end{abstract}

Although the hippocampus has been the major brain structure implicated in the ability of animals to solve spatial problems (Morris, Garrud, \& Rawlins, 1981; O'Keefe \& Nadel, 1978), many studies have also implicated the hippocampal projection targets (such as the septum) in the performance of spatial tasks (Herrmann, Black, Anchel, \& Ellen, 1978; Stahl \& Ellen, 1974). Unfortunately, there have been very few reports of damage to hippocampal projection targets other than the septum. Only relatively recently have other hippocampal targets been systematically examined with respect to their role in the elaboration of spatial behavior.

Recognizing the fact that the hippocampus has extensive reciprocal connections with cortical as well as limbic structures (Swanson, 1981), Kolb, Pittman, Sutherland, and Wishaw (1982) undertook an investigation of the role of the medial frontal cortex in the elaboration of spatial behavior. They compared the effects of medial frontal damage with those resulting from damage to the medial dorsal nucleus of the thalamus. This is a crucial comparison, since not only does the medial frontal cor-

Funding for this project was provided by a National Science and Engineering Research Council grant (837-18) to the first author and a special grant from the Fondation pour la Recherche Médicale et Fondation Fyssen to the second author. The authors wish to express their thanks to Catherine Thinus Blanc for her aid in writing and reviewing this manuscript.

T. Herrmann's mailing address is: Department of Psychology, University of Guelph, Guelph, Ontario, Canada N1G 2W1. tex receive innervation from the hippocampus, but it also receives extensive innervation from the medial dorsal nucleus of the thalamus. Kolb et al. (1982) clearly demonstrated that medial frontal lesions impaired performance on both the Morris (1980) water maze and the Olton (1979) 8-arm maze. Lesions of the medial dorsal nucleus were without effect on these same spatial tasks. It is clear from these findings that the medial dorsal nucleus does not provide information relevant for the performance of spatial behavior.

With both the Morris water maze and the Olton 8-arm maze, it has been shown that spatial behavior is based on the utilization of extramaze stimuli when they are available (Morris, 1980; Olton, 1979). In both of these tasks, the use of such stimuli is all that is required to enable the animals to perform successfully. We have previously shown, however, that the mere use of extramaze stimuli to guide behavior on the Maier 3-table spatial-integration task is not sufficient to allow animals to perform successfully. Merely feeding the animals on a table and then giving them a test trial does not produce successful performance (Herrmann, Bahr, Bremmer, \& Ellen, 1982; Stahl \& Ellen, 1974). Rather, for the animals to be able to perform successfully on the 3-table task, they require a prior exploratory experience over the entire complex of tables and runways.

In addition to this difference between the 3-table task and the water- or radial-maze problems, there are still other differences that are important and of concern. In 
the water-maze task, the animals are required to start from different locations to find the hidden safety platform, which remains in a constant location. When this has been mastered, the platform is moved to another location. Evidence of spatial learning is assumed when the animals swim longest in the area where the platform is or had previously been located. However, ultimately, the animals learn the new location of the platform. In the radial-arm maze, the animals are started from the same location on each trial and they merely have to go in an efficient manner to a fixed number of places. This involves not reentering a previously entered alley. In contrast, in the 3-table spatial integration task, the location of food is different from one day to the next. In addition, the place from which the animals embark on their search is changed daily.

Of further interest is the fact that the very nature of the testing procedures used in both the Morris water maze and the Olton radial maze preclude determining whether the animals are having difficulty in acquiring the relevant spatial information or are simply not able to use such information following experimental brain injury. In contrast, in the multistage 3-table task, it is possible to dissociate these potential differences. For example, Ellen and Weston (1983) have shown that rats with septal damage fail on the test trial of the 3-table task. Although that result was not remarkable or new, a close examination of the task's exploratory phase revealed a plausible explanation of that failure. Ellen and Weston (1983) found that normal animals show a gradual decrease in the number of tables entered over the 15-min exploratory period, whereas septal animals continue to enter tables at a high rate over the entire period. In short, the septal animals, unlike the normals, fail to habituate during the exploratory phase. This finding raised the possibility that the failure by septal animals on the 3-table task was the result of a failure to acquire the spatial information needed to perform successfully.

The problem is thus at hand. To what extent are the deficits seen on the Morris water maze and Olton radial maze following medial frontal lesions the result of the failure to acquire the necessary spatial information or, alternatively, the result of a failure in the ability to use such information. The present experiment addresses this question by examining both exploratory behavior and test performance on the Maier 3-table test.

\section{METHOD}

\section{Subjects}

Eighteen naive male Long-Evans hooded rats, approximately 90 days of age, served as subjects. The rats were individually housed in a temperature-controlled vivarium $\left(20^{\circ} \pm 2^{\circ} \mathrm{C}\right)$ on a 14 -h $(0600$ $2000 \mathrm{~h})$-light and 10-h(2000-0600 h)-dark cycle. The animals' weights ranged from 350 to $410 \mathrm{~g}$ when they were received from the University of Guelph breeding colony. They were maintained on an ad-lib food-and-water schedule until the beginning of the experiment. When the experiment began, the animals continued to receive water ad lib while being food deprived for $23 \mathrm{~h}$ (weight was reduced to an average of $90 \%$ of preexperimental body weight).

\section{Apparatus}

The apparatus and its location within the experimental environment have been previously described (Herrmann et al., 1978) in detail. Basically, it consisted of three unique tables interconnected by a Y-shaped runway system. Obstruction screens at the front of each table prevented the animals from seeing the contents of a table when they were either on the table or at the intersection of the runways.

\section{Surgery and Histology}

Prior to the beginning of training, the animals were assigned randomly to one of two groups: normal controls or medial front lesioned. The animals in the medial frontal group were anesthetized with $55 \mathrm{mg} / \mathrm{kg}$ of pentobarbital (Somnotol) intraperitoneally $10 \mathrm{~min}$ following an injection of $.3 \mathrm{ml}$ atropine sulfate. The animals were also given a subcutaneous injection of lidocaine hydrochloride prior to the midline surgical incision.

The animals were restrained in a Kopf stereotaxic instrument. Rf lesions were produced via a Kopf RFG-4 electrode lowered to $\mathrm{A} / \mathrm{P},+9.5$ and $+11.0, \mathrm{D} / \mathrm{V},+8.0$ and $+9.5, \mathrm{M} / \mathrm{L}, \pm 1$, from stereotaxic zero. The electrode tip was heated to $70^{\circ} \mathrm{C}$ for $15 \mathrm{sec}$. Following completion of the experiment, the animals were sacrificed with a lethal dose of pentobarbital and perfused intracardially with saline followed by $10 \%$ formalin. Prior to sectioning, the brains were removed and stored in formalin. Frozen sections, $40 \mu$ in thickness, were taken with every 10 th section and stained with cresyl violet for the purpose of identifying lesion location and extent.

\section{Procedure}

During the 12-day postoperative period, the animals received daily 10-min handling sessions. During the last 5 days of this period, the animals were placed on a straight runway suspended between two chairs in the experimental room and allowed to run to the end of the runway for food. The animals were given 10 such trials on each of the 5 days.

Testing on the Maier 3-table task took place over the following 24 days. As is typical on this task, the animals received a 15-min daily exploratory period over the entire unbaited apparatus. Following this exploratory experience, the animals were placed on that day's goal, or feeding, table, where they were allowed to eat for 1 min. Following the feeding experience, the animals were individually placed on that day's start table, and their task was to return to the place where they had just been fed. Each day, a different combination of start and feeding tables was used. The animals were allowed $3 \mathrm{~min}$ to leave the start table and reach the goal table. An error was scored when an animal either failed to leave the start table or went to the nonbaited table. In the latter instance, the animal was allowed to self-correct.

\section{RESULTS}

\section{Anatomical Findings}

The rostral medial dorsal lesions shown in Figure 1 removed all midline medial frontal cortex (Leonard, 1972). There was minimal damage to midline cortex posterior to the genu of the corpus callosum.

Inspection of the medial dorsal nucleus of the thalamus revealed subtle bilateral cell loss. Similar cell loss was seen in the ventromedial thalamic region.

\section{Behavioral Findings}

Test trial performance. Figure 2 portrays the test-trial performance for both groups of animals over the course of the 24-day test period. The normal animals performed at the $75 \%$ level over the course of the experiment. The 


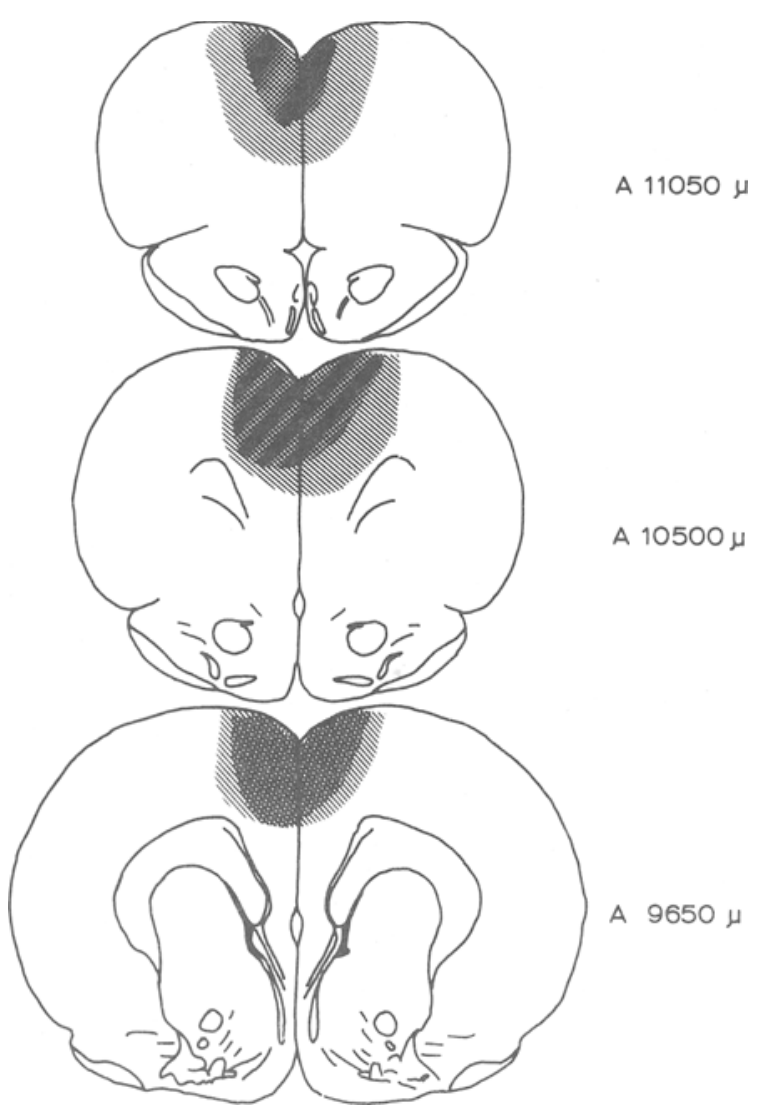

Figure 1. Diagramatic representation (based on König \& Klippel, 1963) of histological results. Drawings present range (maximum $=$ lightly shaded, minimum $=$ darkly shaded) of damage to medial frontal cortex.

medial frontal animals, in contrast, showed performance ranging from $50 \%$ to $65 \%$ correct over the experiment. A repeated measures ANOVA, with surgical treatment as the between-groups measure and blocks of days as the repeated within measure, confirmed these impressions $[\mathrm{F}(1,8)=57.25, \mathrm{p}<.001]$. More importantly, there was a significant interaction between lesion and block of trials $[F(3,24)=3.47, p<.04]$. It is clear from subsequent t-test analysis that this interaction identifies the fact that animals with medial frontal lesions are markedly impaired on the 3-table spatial integration task and that there is no recovery from that impairment over the 24 -day period.

Although the performance of the medial frontal rats tended to fluctuate around the $50 \%$ correct level, this performance was not random. Rather, the medial frontal animals tended to make the same body turn at the intersection of the runways on more than $75 \%$ of the trials (mean $=75.8 \%, \mathrm{SE}= \pm 3.6 \%)$. In contrast, the normal animals did not show the same degree of perseveration. They repeated the same body turn on only $55 \%$ of the trials $($ mean $=55.2 \%, \mathrm{SE}= \pm 1.3 \%)$. This difference was highly significant $[\mathrm{F}(1,16)=25.7, \mathrm{p}<.0001]$.
Exploratory behavior. The exploratory-period behavior was analyzed in detail, with the daily 15 -min period broken down into successive 3-min intervals and the number of tables entered during each successive interval counted. These findings are presented in Figure 3, which shows the changes in table-entry behavior over successive intervals as the course of testing progressed. The data were analyzed by a repeated-measures ANOVA that compared the exploratory behavior of both groups using blocks of trials and intervals as the repeated measures. No difference in the overall amount of exploration by each group was revealed $[\mathrm{F}(1,8)=3.57, \mathrm{p}=\mathrm{n} . \mathrm{s}]$. There was a significant three-way interaction between groups, blocks of trials, and interval $[\mathrm{F}(12,96)=6.29, \mathrm{p}<.001]$, which was examined via subsequent preplanned t-test analysis.

As shown in Figure 3, the pattern of exploration was different for each surgical treatment group. More specifically, it should be noted that normal animals in the first block of six trials showed a decrease in the number of table entries from the first 3-min interval to the last 3min interval. This decrease continued through the 17 th day of testing [i.e., preplanned t-test comparisons reveal significant differences between the initial daily 3-min interval and the final daily interval during the first three blocks of testing; all ts(7) = between 3.14 and 2.25 , p $<.05]$. In contrast, the medial frontal animals entered as many tables during the last $3 \mathrm{~min}$ of the period as they did in the first 3 min during the initial block of trials [t(7) $=1.39, \mathrm{p}=$ n.s.]. However, by the second block of trials, the medial frontal trend had changed. In this second block and for the remaining blocks of trials, medial frontal animals also demonstrated the decreasing tableentry pattern [all ts $(7)=$ between 4.14 and 2.61 , p < $.05]$. This tendency to decrease activity (habituation) has been previously identified (O'Keefe \& Nadel, 1978) as indicative of exploratory, rather than general activity, behavior.

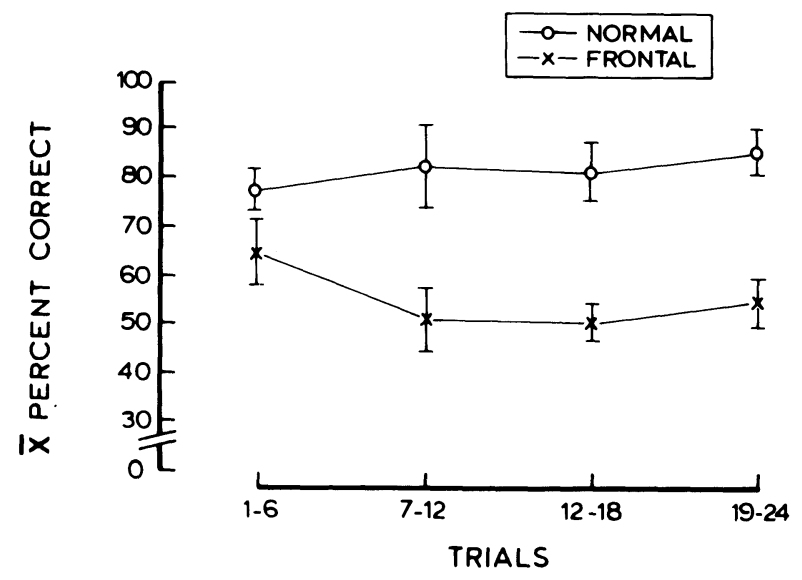

Figure 2. Comparison of the mean (with standard error noted) first choice behavior of normal and medial frontal animals over blocks of six trials. 


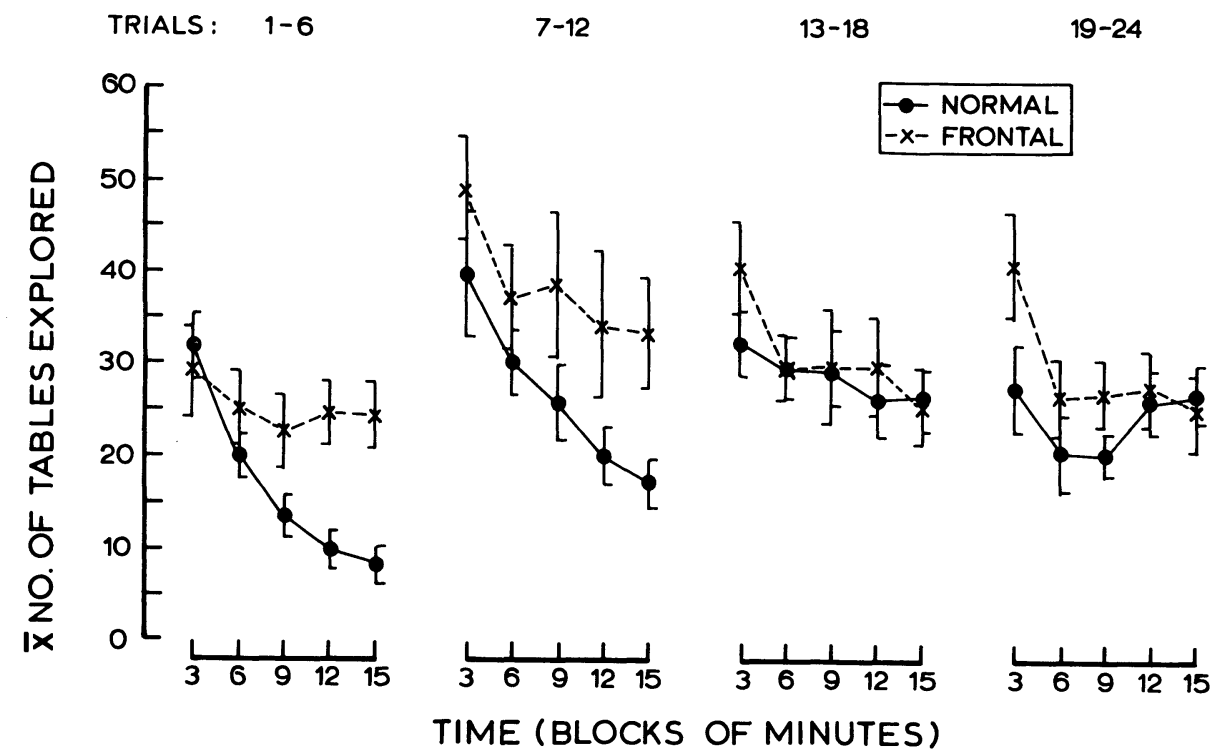

Figure 3. Comparison of the mean (with standard error noted) number of tables entered by normal and medial frontal animals during the successive 3-min intervals of the exploratory period over blocks of six trials.

It should be noted that during the final block of trials, although normal animals did habituate following the first 3 -min interval $[t(7)=2.74, p<.05]$, they increased the number of table entries from the end of the third 3-min interval until the end of the daily exploratory period $[t(7)$ $=3.17, \mathrm{p}<.03$ ].

\section{DISCUSSION}

It is clear from the findings of this experiment that animals with medial frontal lesions fail on the Maier 3table spatial integration task. As is seen in animals with septal damage, medial frontal rats adapt to this task by adopting a consistent body-turn strategy (Herrmann et al., 1978). These findings are also consistent with those of Kolb et al. (1982), who found that the performance of medial frontal animals was deficient on both the Morris water maze and the Olton radial-arm maze. Unlike the Kolb findings, the results of the present study indicate no evidence of improvement in the brain-damaged animals with repeated testing. To the extent that, in both the water maze and the 3-table task, the deficits resulting from medial frontal damage were permanent but in the radial maze the deficit was transitory, the possibility exists that the water maze and the 3-table task reflect the operation of psychological processes different from those involved in radial-maze performance. This hypothesis of course cannot be evaluated within the context of the present experiment.

Of greater interest, perhaps, than the mere failure of the medial frontal rats on the 3-table task are the differences seen in these rats with respect to their behavior during the 15-min exploratory period. It will be recalled that, initially, the overall table entries made by medial frontal animals were constant during the entire 15-min exploratory period. These animals did not begin to decrease their table-entry behavior until the second block of trials. This is in contrast to normal animals, which demonstrated a decrease in table-entry behavior from the very earliest trials. O'Keefe and Nadel (1978) differentiated between exploratory activity and general activity by noting that exploratory activity was evidenced by a decrease from initial levels while general activity remained constant over time. From this perspective, it would seem that normal animals demonstrated exploratory behavior from the very beginning of the experiment, whereas medial frontal animals did not demonstrate exploratory behavior until the second block of six trials.

This finding stands in contrast to the results reported by Ellen and Weston (1983) with respect to septal lesions. Unlike medial frontals, animals with septal lesions fail to demonstrate any decreasing pattern of table-entry behavior during the 15 -min exploratory periods. It might be suspected, then, that the basis of the deficit in septal animals is different from that seen in medial frontals. Although it may be that septal animals fail on the 3-table task because of a failure to learn the spatial relations existing among the tables during the exploratory period, it is clear that such an explanation does not apply to the medial frontals. Rather, it would appear from our data that the medial frontals are able to acquire the requisite information concerning relationships among the tables.

Additionally, normal animals showed an increase in exploratory activity within the 15 -min exploratory period on the final block of trials. A comparable increase was not evidenced by medial frontal animals during this period. It might be suspected that the increased activity of the normal animals could be due to the approaching 
onset of the feeding phase, which would provide incentive motivation. Since the medial frontal animals failed to show an increase in table entries toward the end of the exploratory period, the possibility exists that the medial frontal deficit may derive from a failure of the feeding experience to inform the animal as to the locus and/or availability of food (Herrmann et al., 1982). This would suggest that the medial frontal deficit is related more to the nature of the information available during the feeding experience than to the information obtained during exploration.

\section{REFERENCES}

Ellen, P., \& Weston, S. (1983). Problem solving in the rat: Septal lesion effects on habituation and perseverative tendencies. Physiological Psychology, 11, 112-118.

Herrmann, T., Bahr, E., Bremmer, B., \& Ellen, P. (1982). Problem solving in the rat: Stay vs. shift solutions on the three-table task. Animal Learning \& Behavior, 10, 39-45.

Herrmann, T., Black, A. H., Anchel, H., \& Ellen, P. (1978). Comparisons of septal and fornical lesioned rats' performance on the Maier 3-table reasoning task. Journal of Comparative and Physiological Psychology, 20, 297-302.
Kolb, B., Pittman, K., Sutherland, R. J., \& Wishaw, I. Q. (1982). Localization of the contribution of the medial frontal and dorsomedial thalamic nucleus to spatially guided behavior in the rat. Behavioral Brain Research, 6, 365-378.

König, J. F. R., \& KLIPPEL, R. A. (1963). The rat brain: A stereotaxic atlas. Baltimore: Williams \& Williams.

LEONARD, C. M. (1972). The connections of the dorsomedial nuclei. Brain Behavior and Evolution, 6, 524-542.

MoRris, R. G. M. (1980). Spatial location does not require the presence of local cues. Learning and Motivation, 12, 239-260.

Morris, R. G. M., Garrud, P., \& Rawlins, J. N. P. (1981). Hippocampal ablations cause spatial reference memory deficits in the rat. Society for Neuroscience Abstracts, 7, 237.

O'KEEFE, J., \& NADEL, L. (1978). The hippocampus as a cognitive map. Oxford: Clarendon Press.

Olton, D. S. (1979). Mazes, maps and memory. American Psychologist, 34, 583-596.

Stahl, J. M., \& Ellen, P. (1974). Septal lesions and reasoning performance in the rat. Journal of Comparative and Physiological Psychology, 84, 629-638.

Swanson, L. A. (1981). A direct projection from Ammon's horn to prefrontal cortex in the rat. Brain Research, 217, 150-154.

(Manuscript received June 27, 1984; revision accepted for publication April 20, 1985.) 